\title{
Chain DRL behavior in rats
}

GERALD H. TRUMBULE ${ }^{1}$, RICHARD W. SWITALSKI AND FLORENCE GILBARD UNIVERSITY OF MARYLAND, COLLEGE PARK, MARYLAND

Rats were reinforced on a chain DRL $9 \sec D R L 9 \sec D R L$ 9 sec schedule. Responding in the member closest to primary reinforcement approximated the $9 \mathrm{sec}$ requirement while in the other two members responses were spaced longer than $9 \mathrm{sec}$. A comparison was drawn between amount of food reinforcement and position in a chain schedule. Efficiency ratios, reinforcement rates, and relative frequency of interresponse times were compared as measures of DRL behavior.

It has been demonstrated that a DRL schedule-one which differentially reinforces long inter-response times-is sensitive to some motivational variables (intensity of electrical brain stimulation, Kling \& Beer, 1962; reinforcement size, Beer \& Trumbule, 1965) and insensitive to others (deprivation and progressive satiation, Conrad et al, 1958; liquid deprivation, Mechner \& Guevrekian, 1962). The purpose of the present study was to determine how behavior changes as a function of the distance from primary reinforcement in a chain DRL schedule.

\section{Methed}

The Ss were four naive male albino rats, T1-T4, from the Walter Reed Army Institute of Research rat colony. Their weights ranged from $300-500 \mathrm{~g}$ at the start of the experiment. They were maintained at $85 \%$ of this ad lib weight. Water was available in both the home cage and the experimental chamber.

The Scientific Prototype chamber had a modified telegraph lever at one end and a food cup at the other. The stimuli were three $28 \mathrm{~V}$ lamps with clear jewels mounted $4 \mathrm{~cm}$ above the lever and spaced $6 \mathrm{~cm}$ apart. S1, the stimulus immediately preceding food reinforcement, was to the right, S2 was in the center, and S3 was to the left of the lever. The reinforcer was a $45 \mathrm{mg}$ Noyes rat pellet. The chamber was housed in a sound attenuating ice chest. Programming equipment was located in an adjacent room.

Preliminary training consisted of placing the subject in the chamber overnight with a pellet available for each lever press (CRF). None of the stimuli was lighted. This procedure was repeated nightly until over 300 pellets were received during a single training session.

For the next 10 daily $1 \mathrm{~h}$ sessions only responses separated by at least $9 \mathrm{sec}$ were reinforced (DRL $9 \mathrm{sec}$ ). S1 was lighted throughout these sessions. During the following 10 sessions S2 was lighted initially. Responses separated by at least $9 \mathrm{sec}$ in the presence of S2 turned S2 off and lighted $\$ 1$. Responses separated by at least 9 sec in the presence of $S 1$ were reinforced with food (chain DRL $9 \mathrm{sec}$, DRL $9 \mathrm{sec}$ ). For the remaining 30 days of the experiment, a three-member chain (DRL $9 \mathrm{sec}$, DRL $9 \mathrm{sec}$, DRL $9 \mathrm{sec}$ ) was in effect. Appropriately spaced responses in the presence of
S3 turned S3 off and lighted S2, and so on. Responses less than 9 sec apart did not change the stimuli or deliver a pellet, but only reset the 9 sec contingency, thereby requiring the next response to occur at least 9 sec later in order to change stimuli or to deliver a pellet.

\section{Resulis and Discussion}

All computations are based on the means of the last 10 days of performance. The efficiency ratios (reinforced responses/total responses) in column A (solid line) of Fig. 1 demonstrate the differential performance in the three members of the chain. Most of the responses in the presence of $S 3$ were reinforced (by the changing of the stimuli) while in $S 2$ and $S 1$ the ratios were considerably reduced. Thus, the "efficiency" of the Ss' behavior decreased as the primary reinforcement was approached. These ratios show a decrease of as much as $77 \%$ from $\mathrm{S} 3$ to $\mathrm{S} 1$.

Beer \& Trumbule (1965), using a two-lever (A-B DRL) situation, found similar differential performance using one, two, or four pellets, with four pellets producing the lowest efficiency ratio (highest response rate). Their procedure, however, eliminated the possibility of "bursting" (rapid responding). Recomputation of the present data excluding these bursts of response (any response which follows a response by less than $3 \mathrm{sec}$ ) resulted in a differential increase in the efficiency ratios with S3 changing the least and S2 and S1 considerably more, as shown in the dotted lines in column A. These

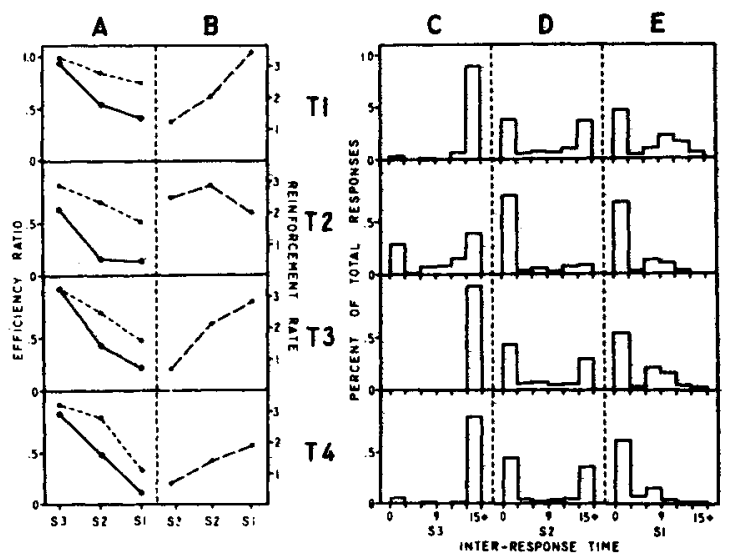

Fig. 1. Data is computed from the mean of the last 10 days of performance. Column A shows the efficiency ratios (reinforced responses) total responses) including responses in the $\mathbf{0 - 3} \mathrm{sec}$ interval (solid lines) and excluding these responses (dotted lines) for the three members of the chain, S3, S2, and S1. Column B shows the reinforcement rates (reinforcements per minute) for the three members. Columns C, D, and E show the relative frequency distribution histograms for the three members. Note that reinforcement availability is indicated by the $9 \mathrm{sec}$ mark in the histograms. 
recomputed efficiency ratios are very similar to those shown in Beer \& Trumbule.

The rationale for this recomputation is apparent upon examination of the inter-response time (IRT) distributions in columns C, D and E of Fig. 1. Note that the percentage of responses in the 0-3 sec interval increased steadily from S3 to S1. Thus, a good proportion of the decrease in efficiency from S3 to S1 was due to the increase in bursting. The sensitivity of the burst of responses to position in the chain may deserve further attention as a measure of motivational level in DRL schedules.

These same IRT distributions (columns C, D and E of Fig. 1) indicated that there was very little "timing" behavior in S3 and S2. With the possible exception of $T 2$, almost all of the responses in S3 occurred after $15 \mathrm{sec}$ had elapsed (mean across subjects $=78 \%$ of total responses). This may be accounted for, in part, by the eating and grooming behavior which normally takes place after the delivery of a pellet. However, even in S2 many of the responses occurred after pauses of greater than $15 \mathrm{sec}$ (mean across subjects $=28 \%$ of total responses).

It was only in S1 that the "typical" IRT distribution began to appear with a second mode approximating the availability of reinforcement $(9 \mathrm{sec}$ ) (Wilson \& Keller, 1953). Thus, the IRT distributions show that, although fewer of the responses were reinforced in S1 than in S3 (as indicated by the efficiency ratios, column A), the behavior in $\mathrm{S1}$ was more appropriate in terms of the reinforcement contingencies than in S3. Further evidence of this appropriateness is shown by the reinforcement rates ( reinforcements per minute) in column $B$ of Fig. 1. With the exception of T2, S3 had the lowest rate of reinforcement and $S 1$ the highest. Thus, the reinforcement rate gives an opposite impression from the efficiency ratio, i.e., the behavior seemed to become more appropriate as the primary reinforcement was approached.

The response rates (responses per minute) under the three stimuli were as follows: Subject $T 1, S 3=1.4$, $\mathrm{S} 2=3.7, \mathrm{~S} 1=8.7 ; \mathrm{T} 2, \mathrm{~S} 3=4.9, \mathrm{~S} 2=16.0, \mathrm{~S} 1=17.0$; $\mathrm{T} 3, \mathrm{~S} 3=.7, \mathrm{~S} 2=4.5, \mathrm{~S} 1=12.5$; and $\mathrm{T} 4, \mathrm{~S} 3=1.0, \mathrm{~S} 2=3.3$, $S 1=15.7$. The total time spent in the presence of the stimuli decreased as primary reinforcement was approached while the number of responses increased from
S3 to S1. Thus the rates were very low in S3 and much higher in $\mathrm{S} 1$.

In their study comparing tandem DRL schedules with chain DRL schedules, Thomas \& Stubbs (1967) point out that the lower response rates in the initial (S3) component of a chain DRL schedule may be due to the discriminative function of that stimulus in its association with "non-reinforcement." They conclude that this lower rate may "obscure any possible rate-enhancing conditioned reinforcing function of stimuli associated with the next component." However, in view of the similarity between the present data and the data of Beer \& Trumbule, it seems more likely that the decrement in rate with distance from primary reinforcement is a manifestation of the decreasing effectiveness of the conditioned reinforcer (Kelleher \& Gollub, 1962). Thus, just as one pellet produced lower rates and higher efficiency ratios than did four pellets, S3 produced lower rates and higher efficiency ratios than did $S 1$.

From the above, three conclusions may be drawn: (1) Behavior maintained under a DRL schedule of reinforcement is sensitive to position in a three-member chain; (2) the more distant the primary reinforcer, the less effective the conditioned reinforcer; and (3) the appropriateness of behavior with respect to the schedule contingencies must be considered from more than one standpoint as any one of the four measures used here might alone have given a spurious impression.

\section{References}

BEER, B., \& TRUMBULE, G. H. Timing behavior as a function of amount of reinforcement. Psychon. Sci, 1965, 2, 71-72.

CONRAD, D. G., SIDMAN, M., \& HERRNSTEIN, R. J. The effects of deprivation upon temporally spaced responding. $J$. exp. A nal Behav., 1957, 1, 59-65.

KELLEHER, R. T., \& GOLLUB, L. R. A review of positive conditioned reinforcement. J. exp. Anal Behav., 1962, 5, 543-597.

KLING, J. W., \& BEER, B. Timing behavior as a function of current and locus of intracranial stimulation. East. Psychol Assoc. Meet., 1962.

MECHNER, F., \& GUEVREKIAN, L. Effects of deprivation upon counting and timing in rats. J. exp. Anal Behav., 1962, 4, 463-467.

THOMAS, J. R., \& STUBBS, A. Stimulus control of temporally spaced responding in second-order schedules. J. exp. Anal Behav., 1967, 10, 175-183,

WILSON, M. P., \& KELLER, F. S. On the selective reinforcement of spaced responses. J. comp. physiol Psychol, 1953, 46, 190-193. Note

1. Now at Psychology Department, 106 College Hall, University of Pennsylvania, Philadelphia, Pa. 19104. 